\title{
A mathematical approach to medical diagnosis via Pythagorean fuzzy soft TOPSIS, VIKOR and generalized aggregation operators
}

\author{
Khalid Naeem ${ }^{1}$ (D) Muhammad Riaz $^{2}$ - Faruk Karaaslan ${ }^{3}$
}

Received: 20 December 2020 / Accepted: 3 July 2021 / Published online: 16 July 2021

(c) The Author(s) 2021

\begin{abstract}
Hepatitis is a therapeutic disorder caused by the inflammation/infection of liver and regarded as the existence of cells causing inflammation in the tissues of body parts. Hepatitis is deliberated as a lethal disease worldwide, especially in developing countries mainly due to contaminated drinking water, unhygienic sanitary conditions and careless blood transfusion. This infection is basically considered as viral infection even though this sort of liver infection can also take place due to autoimmune, toxin, medications, unprotected physical relations, drugs and alcohol. Many approaches of identifying viral hepatitis have been sought so for, which include physical inspection, liver function tests (LFTs), liver surgery (biopsy), imaging studies such as sonogram or CT scan, ultrasound, blood tests, viral serology panel, DNA test, and viral antibody testing. In this article, we propose mathematical analysis of viral hepatitis types using Pythagorean fuzzy soft sets (PFSSs) via TOPSIS (Technique for Order of Preference by Similarity to Ideal Solution), VIKOR (Vise Kriterijumska Optimizacija Kompromisno Resenje) and generalized aggregation operators models and show that all the three techniques render the same optimal choice. We also present a commentary yielding comparison between the three techniques considering their structure of evaluation.
\end{abstract}

Keywords Pythagorean fuzzy soft sets · TOPSIS · VIKOR · Generalized aggregation operators · Medical diagnosis

\section{Introduction}

Comprehensive decision making is a very complex task, especially when one faces ever-changing situations and information. Multi-criteria group decision making (MCGDM) refers to making judgment with more than one decision maker (DM) in the presence of several principles. Problems in everyday life call for bearing in mind multifaceted structure of norms which affects solutions and the final conclusion.

Khalid Naeem

khalidnaeem333@gmail.com

Muhammad Riaz

mriaz.math@pu.edu.pk

Faruk Karaaslan

fkaraaslan@karatekin.edu.tr

1 Department of Mathematics and Statistics, The University of Lahore, Lahore, Pakistan

2 Department of Mathematics, University of the Punjab, Lahore, Pakistan

3 Department of Mathematics, Çankırı Karatekin University, 18100 Çankırı, Turkey
MCGDM models arrange for a platform for assessing the criteria and have some features. Some of them are:

- A criteria may be a trait or spatial data.

- The criteria may be conflicting, i.e., the intra-conflicts and intra-similarities.

- Each criterion may be measured in different units.

- The process of computing relative weights for each criterion is entirely flexible.

MCGDM consists of different types of ways and means to provide decision with manifold and conflicting criteria. TOPSIS and VIKOR are the extensively employed techniques to handle such situations. The foundation of TOPSIS is laid on the principle that we try to explore the possibly finest choice from the available ones which is at the tiniest distance from the so-called "positive ideal solution" (PIS) and farthest away from "negative ideal solution" (NIS). PIS signifies maximized advantage criteria and minimized cost criteria. Thus, in the other sense, we can say that NIS denotes minimized benefit criteria and maximized cost criteria. TOPSIS is a simple multi-criteria decision analysis methodology used in many practical problems. On the other hand, VIKOR is 
an MCGDM scheme to decide the compromise ranking and compromise solution by means of agreed criteria weights. VIKOR concentrates upon ranking/grading and opting for a collection of alternatives by multiple criteria ranking index established on evaluating the aloofness from the idyllic solution. The compromise ranking list may be determined by computing the nearness of each available choice from the supreme solution.

The initiation of fuzzy sets (FSs) by Zadeh [32] in 1965 was like a great achievement and a point of glee in the world of mathematicians indulged in logic and set theory. Getting motivated by the initiation of FSs, Atanassov [14] involved another parameter, traditionally acknowledged as the dissociation or non-membership grade, in FSs and titled this innovative structure as an intuitionistic fuzzy set (IFS). In the last decade of the twentieth century, Molodstov [17] brought to canvass the theory of soft sets (SSs) a theory that acquiescently labels a number of characteristics (usually called attributes or characteristics) for clarifying and scrutinizing a problem comprising vagueness, ambiguity, obscurity and uncertainty. In his paper, Molodstov also presented some worthwhile practical implementations from daily life. Maji et al. [16] defined the concept of intuitionistic fuzzy soft sets. Yager [29-31] generalized the concepts of IFSs to Pythagorean fuzzy sets (PFSs). Peng et al. [23], and Naeem et al. [19] presented some characteristics of Pythagorean fuzzy soft sets (PFSSs) and studied the related results. Later, Guleria and Bajaj [12] presented matrix form to represent PFSSs.

Çağman et al. [5], by merging fuzzy and soft sets, unfolded a hybrid structure acknowledged as fuzzy soft set theory. Davvaz and Sadrabadi [6] furnished a practical implementation of IFSs in medicinal science. Garg and Arora [9-11] introduced various decision-making methodologies by making use of aggregation operators. Wang and Elhag [27] presented fuzzy TOPSIS method with application to bridge risk management. Eraslan and Karaaslan [8] presented a TOPSIS based group decision-making method under fuzzy soft environment. Zhang and $\mathrm{Xu}$ [33] presented an extension of TOPSIS in MCDM with the help of PFSs. Ratnaparkhi et al. [7] explored a novel entropy-based weighted attribute selection in enhanced multi-criteria decision making using fuzzy TOPSIS model for hesitant fuzzy rough environment. Hwang and Yoon [13] rendered multiple attribute decision-making methods and applications. Kalkan et al. [14] compared the ranking results obtained by TOPSIS and VIKOR methods. Opricovic and Tzeng [21,22] studied comparative analysis of TOPSIS and VIKOR methods. Salabun et al. [25] undertook an attempt to benchmark selected Multi-Criteria Decision Analysis (MCDA) methods comprising TOPSIS, VIKOR, COPRAS, and PROMETHEE II. Shekhovtsov and Salabun [26] presented a comparative case study of the VIKOR and TOPSIS rankings similarity. Naeem et al. [18-20] presented diverse decision making techniques on extension of Pythagorean fuzzy sets with their applications.

The notions of PFSSs and IFSSs, off course too, depend not merely upon membership degree, but also on its counterpart the dissociation grade of the attribute under discussion. A large number of decision-making problems (DMPs) tackled via different techniques depend only on membership grades of the parameter. By keeping in view the values of dissociation grades of the parameter, a more precise and truthful decision can be achieved. This inspires an allembracing study decision making through PFSSs in DMPs. For example, if a person is experiencing abnormal sleep, then by allocating both values, i.e., membership and nonmembership, it becomes easier to categorize whether the person is hypertensive or not. Thus, the modus operandi proposed in this article enables to alarm the patient about his defenselessness of becoming hypertensive.

The motivation and primary objectives of this article are outlined by the following facts:

- Pythagorean fuzzy soft sets, which are a generalization of intuitionistic fuzzy and fuzzy soft sets, are a more useful tool in decision-making problems since they are a parameterized family of Pythagorean fuzzy sets. Also, TOPSIS and VIKOR methods are two important methods for decision-making problems. In this study, by combining the modeling advantages of Pythagorean fuzzy flexible sets and the advantages of TOPSIS and VIKOR methods, we have developed two methods in the Pythagorean fuzzy soft environment.

- Fuzzy soft sets and intuitionistic fuzzy soft sets have applications in many fields. However, fuzzy sets and intuitionistic fuzzy sets have some limitations in modeling problems. Pythagorean fuzzy soft sets offer an approach away from these limitations and give more precise and accurate results in applications. Therefore, in this article, two applications based on TOPSIS and VIKOR methods related to Hepatitis are discussed in the Pythagorean fuzzy soft environment.

Following are the contributions of this article:

- TOPSIS and VIKOR methods are developed in Pythagorean fuzzy soft environment and given applications of them for medical diagnosis of Hepatitis.

- A medical diagnosis method is developed based on generalized PFS aggregation operators and is given an application in medical diagnosis of Hepatitis.

- The consistency of the proposed methods is shown by making a comparison with each other and with other methods in the literature. 
The rest of the article is managed, for smooth conception, as follows: in the next section, we give replication of momentary but ample definitions of different sets. We first present different types of hepatitis along with their symptoms and causes of eruption of that type, in the third section of this article. Afterwards, we use fuzzy weights for the linguistic terms that are to be employed in mathematical computations. We propose, in the same section, PFS TOPSIS Algorithm and employ this Algorithm using hypothetical data in diagnosing which of the patients under consideration is most affected one and the preference order using the said Algorithm. We propose second Algorithm (VIKOR) in the fourth section, and apply it on the same problem of the third section and generate preference ranking using the technique explained in that section. We propose third Algorithm (Generalized PFS aggregation method) in the fifth section and once again employed the proposed algorithm on the same problem discussed via Algorithm 1 and 2. In the sixth section, we give commentary on the difference, comparison and similarities among the three techniques and give opinion about the more suitable technique through solid reasoning. We also compare the optimal choice using some existing techniques which validates the proposed techniques. Finally, in the last section, we finish with a concrete conclusion and some prospect recommendations.

\section{Preliminaries}

In the present unit, we kindle memories of some introductory notions of different genera of sets with brevity, that will assist in comprehending the forthcoming part of the study.

Definition 2.1 [32] A collection of ordered pairs $\left(\hbar, \tau_{\mathcal{F}}(\hbar)\right)$, $\hbar$ being an element of the underlying universe $X$ and $\tau_{\mathcal{F}}$ (the affiliation, association or membership function) is a welldefined map, that drives members of $X$ to $[0,1]$, is entitled as a fuzzy set $(\mathrm{FS}) \mathcal{F}$ over $X$. In other words

$\tau(\hbar)= \begin{cases}1, & \text { if } \hbar \in \mathcal{F} \\ 0, & \text { if } \hbar \notin \mathcal{F} \\ 0,1[, & \text { if } \hbar \text { is partially in } \mathcal{F}\end{cases}$

The assembly of all FSs over $X$ is usually delineated as $\mathcal{F}(X)$.

Definition 2.2 [17] Supposing $E$ a non-empty assembly of traits (technically known as attributes) with $A \subseteq E$. A pair of the form $(\Upsilon, A)$, where $\Upsilon: A \rightarrow 2^{X}$ is a mapping, is termed as soft set (SS) over $X$. Thus, in set-builder form, we write

$(\Upsilon, A)=\left\{(\Theta, \Upsilon(\Theta)): \Theta \in A, \Upsilon(\Theta) \in 2^{X}\right\}$

The collection $(\Upsilon, A)$ is also expressed as $\Upsilon_{A}$.
Definition 2.3 [5] Take $X$ to be an underlying classical set and $A$ the sub-collection of attributes' set $E$. A collection of ordered doublets

$\Psi_{A}=\left\{\left(\Theta, \psi_{A}(\Theta)\right): \Theta \in E, \psi_{A} \in \mathcal{F}(X)\right\}$

is accredited as fuzzy soft set (FSS) over $X$.

The aggregate of all FSSs over $X$ is designated as $F S(X)$.

Definition 2.4 [1-3] An intuitionistic fuzzy set (IFS) over the classical set $X$ is a collection containing ordered triplets like $(\hbar, \tau(\hbar), o(\hbar))$, where $\hbar$ is a member of $X$ and $\tau, o$ are mappings restricted to obey the requirement that sum of their values must not surpass unity, acknowledged respectively as the affiliation and dissociation grades, that drive members of $X$ to $[0,1]$.

The assembly of all IFSs over $X$ is designated as $\operatorname{IF}^{X}$.

Definition 2.5 [16] Understand that $X$ is a crisp set endowed with $A$ as the sub-family of attributes' set $E$. A multi-valued mapping $\psi$ that sends elements of $A$ to $\mathrm{IF}^{X}$ is termed as an intuitionistic fuzzy soft set (IFSS) over $X$ and has representation as $(\psi, A)$ or $\psi_{A}$.

Definition 2.6 [29-31] A Pythagorean fuzzy set, abbreviated as PFS, is a family

$$
P=\left\{<\hbar, \tau_{P}(\hbar), o_{P}(\hbar)>: \hbar \in X\right\}
$$

where $\tau_{P}$ and $o_{P}$ are mappings from a set $X$ to $[0,1]$ following the constraint $0 \leq \tau_{P}^{2}(\hbar)+o_{P}^{2}(\hbar) \leq 1$, representing correspondingly the affiliation and dissociation grades of $\hbar \in X$ to $P$. The ordered pair $p=\left(\tau_{p}, o_{p}\right)$ is known as Pythagorean fuzzy number (PFN). The quantity $\exists(\hbar)=\sqrt{1-\tau^{2}(\hbar)-o^{2}(\hbar)}$ is accredited as the hesitation margin.

Definition $2.7[12,19,23]$ Let $X$ and $E$ have the usual meanings. Assume that $A \sqsubseteq E$ and $\mathrm{PF}^{X}$ represents the class of all PFSs over $X$. A Pythagorean fuzzy soft set (PFSS) on $X$ is denoted as $(\Upsilon, A)$ or $\Upsilon_{A}$, where $\Upsilon: A \rightarrow \mathrm{PF}^{X}$ is a mapping, and is defined by

$$
\begin{aligned}
(\Upsilon, A) & =\left\{\left(\Theta,\left\{\hbar, \tau_{\Upsilon_{A}}(\hbar), o \Upsilon_{A}(\hbar)\right\}\right): \Theta \in A, \hbar \in X\right\} \\
& =\left\{\left(\Theta,\left\{\frac{\hbar}{\left(\Upsilon_{\Upsilon_{A}}(\hbar), o \Upsilon_{A}(\hbar)\right)}\right\}\right): \Theta \in A, \hbar \in X\right\} \\
& =\left\{\left(\Theta,\left\{\frac{\left(\tau_{\Upsilon_{A}}(\hbar), o \Upsilon_{A}(\hbar)\right)}{\hbar}\right\}\right): \Theta \in A, \hbar \in X\right\}
\end{aligned}
$$

where $\tau_{\Upsilon_{A}}$ and $o \Upsilon_{A}$ are well-defined maps that drag elements of $X$ to $[0,1]$ along with the property that sum of their squared values should not go above unity.

If we write $\tau_{i j}=\tau_{\Upsilon_{A}}\left(\Theta_{j}\right)\left(\hbar_{i}\right)$ and $o_{i j}=o_{\Upsilon_{A}}\left(\Theta_{j}\right)\left(\hbar_{i}\right)$ where $i$ and $j$ run, respectively, from 1 to $m$ and from 1 to 
Table 1 Tabular representation of PFSS $\Upsilon_{A}$

\begin{tabular}{ccccc}
\hline$\Upsilon_{A}$ & $\Theta_{1}$ & $\Theta_{2}$ & $\cdots$ & $\Theta_{n}$ \\
\hline$\hbar_{1}$ & $\left(\tau_{11}, o_{11}\right)$ & $\left(\tau_{12}, o_{12}\right)$ & $\ldots$ & $\left(\tau_{1 n}, o_{1 n}\right)$ \\
$\hbar_{2}$ & $\left(\tau_{21}, o_{21}\right)$ & $\left(\tau_{22}, o_{22}\right)$ & $\ldots$ & $\left(\tau_{2 n}, o_{2 n}\right)$ \\
$\vdots$ & $\vdots$ & $\vdots$ & $\ddots$ & $\vdots$ \\
$\hbar_{m}$ & $\left(\tau_{m 1}, o_{m 1}\right)$ & $\left(\tau_{m 2}, o_{m 2}\right)$ & $\cdots$ & $\left(\tau_{m n}, o_{m n}\right)$ \\
\hline
\end{tabular}

$n$ then the PFSS $\Upsilon_{A}$ may be represented in tabular form as shown in Table 1.

Guleria and Bajaj [12] proposed a fascinating way of representing the above tabular form in matrix form as

$$
\begin{aligned}
(\Upsilon, A)= & {\left[\left(\tau_{i j}, o_{i j}\right)\right]_{m \times n} } \\
= & \left(\begin{array}{cccc}
\left(\tau_{11}, o_{11}\right) & \left(\tau_{12}, o_{12}\right) & \cdots & \left(\tau_{1 n}, o_{1 n}\right) \\
\left(\tau_{21}, o_{21}\right) & \left(\tau_{22}, o_{22}\right) & \cdots & \left(\tau_{2 n}, o_{2 n}\right) \\
\vdots & \vdots & \ddots & \vdots \\
\left(\tau_{m 1}, o_{m 1}\right) & \left(\tau_{m 2}, o_{m 2}\right) & \cdots & \left(\tau_{m n}, o_{m n}\right)
\end{array}\right)
\end{aligned}
$$

and named it a Pythagorean fuzzy soft matrix (PFS matrix).

\section{Medical diagnosis using PFS TOPSIS}

As a model, in this unit, we first talk over different categories of Hepatitis by giving a short but comprehensive detail of this fatal disease covering its types and symptoms of each type and then employ proposed TOPSIS method to diagnose the most affected person.

\section{Case study}

The word hepatitis is compound of "hepa" meaning liver and "titis" which stands for inflammation. Hepatitis is basically inflammation of the liver, which is the prime body part, serving store energy, digesting foodstuff, and eliminating venomous ingredients from the body. Its major cause is liver injury. There are five viruses that normally communicate a disease to the liver, named using the first five letters of the English alphabet, i.e., from A to E. What makes epidemiologic hepatitis bewildering is that each one of these viruses originates a marginally unalike sickness and has a dissimilar style of thinning out. More or less of these viral toxicities can give rise to critical, long-lasting, or both kinds of hepatitis (Fig. 1).

Hepatitis A: Hepatitis A is a vaccine-preventable and extremely communicable liver septicity caused by hepatitis A virus. This virus is one of a number of categories of hepatitis germs that grounds irritation/inflammation and upset the liver's capability to function properly. Root causes of hepatitis A are intaking adulterated foodstuff or liquid, or close interaction with an diseased individual or object. Trifling instances of hepatitis A don't call for serious medical treatment and are commonly recuperated absolutely with no perpetual liver impairment. Emblems and warning signs of Hepatitis A may consist of tiredness, intestinal irritation, loss of hungriness, dark urine, abrupt nausea and vomiting, mud-colored bowel movements, inferior fever, jaundice, joint discomfort and extreme itching.

Hepatitis B: This kind is a severe liver septicity caused due to hepatitis B virus, clinically termed as HBV. For more or less folks, this infection turns out to be lingering and persists for more than half an year. Having prolonged hepatitis $\mathrm{B}$ escalates menace of developing liver cancer, liver failure or cirrhosis - an ailment that undyingly scars of the liver. Most of the adults having hepatitis B get well completely, even if they have severe signs and symptoms. Babies and kids are more probable to produce a chronic hepatitis B impurity. Symptoms of this infection vary from modest to serious. They normally seem around 1-4 months after someone have been tainted, even though he/she could witness them as timely as two weeks post-infection. Some individuals, especially teenagers, may not have any symptoms. Common ways that HBV can spread are fleshly contact, sharing of needles/syringes, unintentional needle sticks, and mother to newly born infant. The signs of this sort of virus may include tiredness and weakness, nausea and vomiting, abdominal discomfort, loss of appetite, dark urine, fever, jaundice, and increasing level of discomfort in joints (Fig. 2).

Hepatitis C: It is an epidemiologic infection causing liver disorder, sometimes giving rise to grave liver damage. $\mathrm{HCV}$ (hepatitis $\mathrm{C}$ virus) generally blowouts via infected blood. Nowadays, chronic HCV is normally remediable with oral medications taken daily for 2 months to half an year. Almost half of people infected with $\mathrm{HCV}$ are not aware that they are disease-ridden, primarily because they have no visible symptoms, which can take decades to come out. Globally, $\mathrm{HCV}$ occurs in a number of distinctive varieties, branded as genotypes. The symptoms of hepatitis $\mathrm{C}$ include fatigue, itchy skin, dark-colored urine, hepatic encephalopathy, jaundice, bleeding easily, staining effortlessly, legs-swelling, poor appetite, weight loss, ascites and spider angiomas.

Hepatitis D: It is also reckoned as hepatitis delta virus (HDV). This sort of virus is an infection causing liver to be swelled resulting in weakening of liver functionality and cause long-lasting liver issues, the most fatal of which include liver scarring and cancer. Dissimilar to other forms, this type of virus cannot be contracted on its own. It infects individuals who are already infected with HBV. HDV may be acute/critical or long lasting. Acute hepatitis D occurs abruptly and generally causes more serious symptoms. It may 
Fig. 1 Hepatitis virus and human body

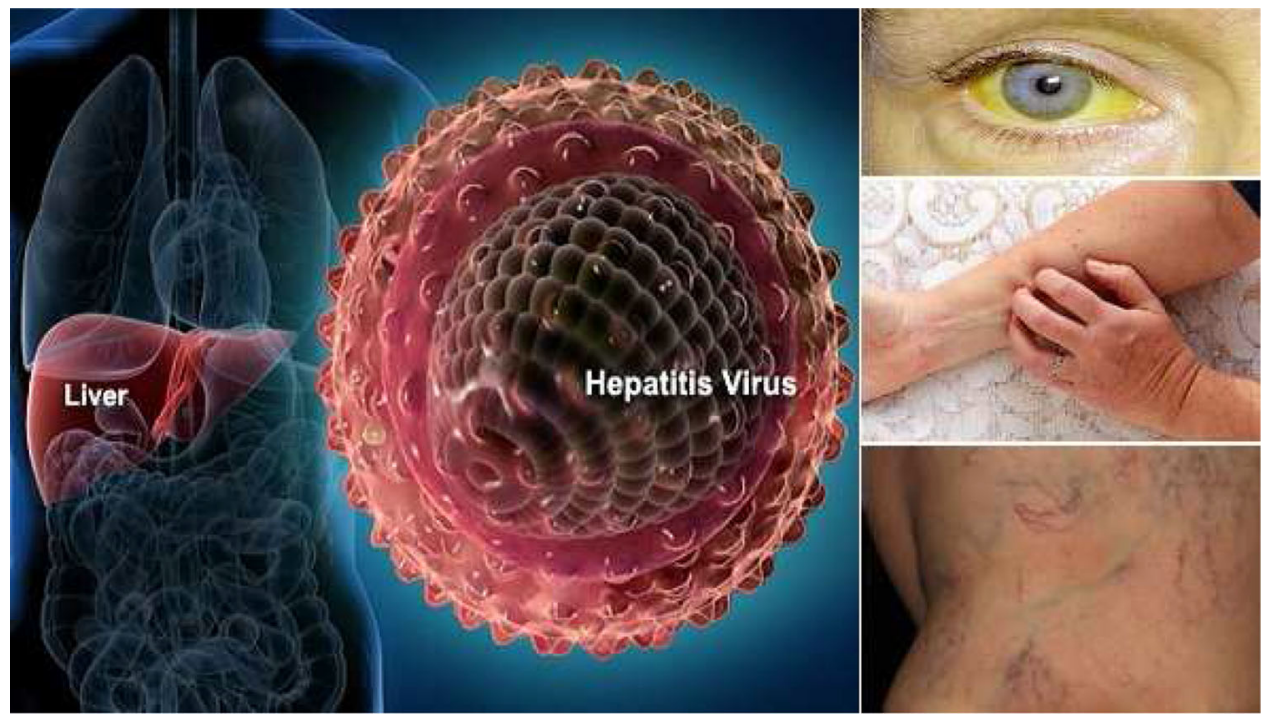

Hepatitis E: Hepatitis E blowouts in several manners. The most commonly ways are using poor-quality drinking liquids and undercooked meat that is contaminated with feces. Fecal matter from human beings or livestock has the potential to impure water, that results ultimately in spread of virus. This type is more prevailing in under-developed territories with substandard quality of water, specifically in populous territories. Pregnant women infected with hepatitis E may also become host of the virus and transferring it to their progeny. In addition to these cases, it is infrequent for individuals to transport this infection to other people. The symptoms of this sort of infection can show a discrepancy. For example, some infected individuals feel no warning signs at all. The other possibility is that the symptoms may be so mild and hardly noticeable. Such situations are more risky and dangerous. In some cases, however, it is observed that some infected individuals experience a few different symptoms that usually appear in 15-60 days after exposure to the virus. Possible symptoms of hepatitis E include poor appetite, fatigue, vomiting, fever, upper abdominal pain, nausea, light/clay-colored stool, jaundice, and dark urine.

For making comprehensive, unanimous and intelligent decisions, we utilize the well-acknowledged technique "TOPSIS" for picking the unbeatable choice from the view point of so-called compromise solution having the quality that the selected solution is neighboring to the idyllic solution and farthest away from the worst solution, i.e., the negative ideal solution.

We exhibit, in this section, how TOPSIS may be employed under Pythagorean fuzzy soft environment. At first, we shall expand TOPSIS to PFSSs and later, apply it to handle a problem from life sciences.

We begin by elaborating the suggested technique stage by stage as below: joint pain (Fig. 3). 
Fig. 3 Healthy and infected human livers
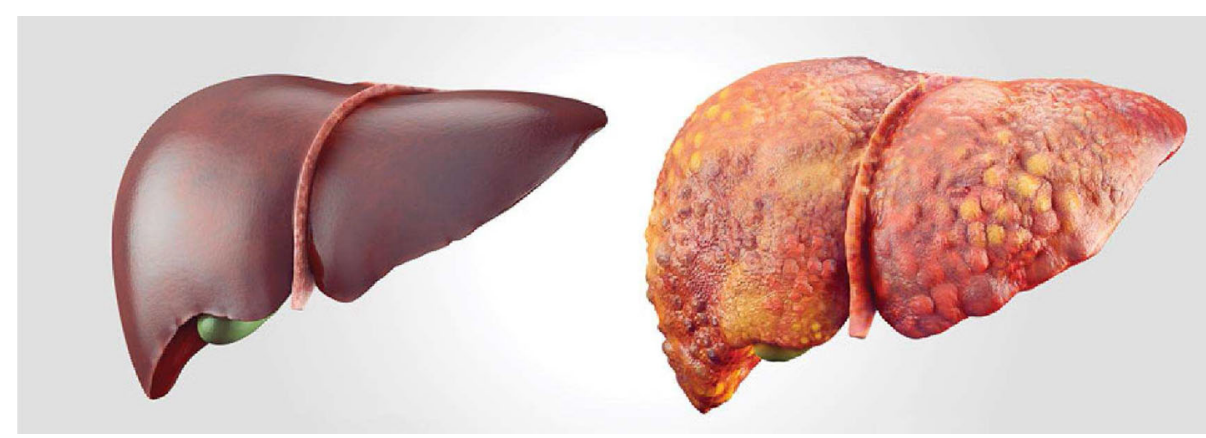

Table 2 Phonological terms for assessing choices

\begin{tabular}{ll}
\hline Phonological terms & Fuzzy weights \\
\hline Stage 0: Healthy liver (S0) & 0.10 \\
Stage 1: Beginning of liver damage (S1) & 0.30 \\
Stage 2: Moderate liver damage (S2) & 0.50 \\
Stage 3: Significant liver damage (S3) & 0.70 \\
Stage 4: Severe liver damage (S4) & 0.90
\end{tabular}

\section{Algorithm 1 (PFS TOPSIS)}

Step 1: Pinpoint the problem: Assume that the team of experts is $E=\left\{\mathcal{D}_{i}\right\}$, the collection of patients under investigation is $V=\left\{\ddot{\rho}_{i}\right\}$ and $D=\left\{\eta_{i}\right\}$ is the family of criteria/parameters, where $i \in \mathbb{N}$.

Step 2: If $w_{i j}$ denotes the weight allocated by $\mathcal{D}_{i}$ to $\eta_{j}$ keeping in view the linguistic variables given in Table 2 , construct weighted parameter matrix $\mathcal{P}=\left[w_{i j}\right]_{l \times m}$.

Step 3: Normalize the weighted parameter matrix $\mathcal{P}$, obtained at Step 2 above. We don't need to separate the parameters as benefit and cost parameter. Therefore, we use the following normalized method to transform cost parameter to the benefit parameter. Normalized values are shown as a matrix denoted by $\hat{N}=\left[\hat{n}_{i j}\right]_{l \times m}$, where $\hat{n}_{i j}=\frac{w_{i j}}{\sqrt{\Sigma_{i=1}^{l} w_{i j}^{2}}}$ and acquire the weight vector $\mathcal{W}=\left(\mathfrak{w}_{j}: j=1,2, \ldots, m\right)$, where $\mathfrak{w}_{j}=\frac{\sum_{i=1}^{l} \hat{n}_{i j}}{l \sum_{k=1}^{m} \hat{n}_{i k}}$

Step 4: Construct PFS decision matrix $D_{i}=\left[\rho_{j k}^{i}\right]_{l \times m}$, where $\rho_{j k}^{i}=\left(\tau_{j k}^{i}, o_{j k}^{i}\right)$. Then obtain the mean proportional matrix

$$
\begin{aligned}
A & =\sqrt[n]{D_{1} D_{2} \ldots D_{n}}=\left[\dot{\rho}_{j k}\right]_{l \times m} \\
& =\left[\left(\sqrt[n]{\left.\left.\prod_{i=1}^{n} \tau_{j k}^{i}, \sqrt[n]{\prod_{i=1}^{n} o_{j k}^{i}}\right)\right]_{l \times m}}\right.\right.
\end{aligned}
$$

Step 5: Compute weighted PFS decision matrix $B=$ $\left[\ddot{\rho}_{j k}\right]_{l \times m}$, where $\ddot{\rho}_{j k}=\mathfrak{w}_{k} \times \dot{\rho}_{j k}=\left(\tau_{j k}, o_{j k}\right)$.
Step 6: Attain PFSV-PIS (PFS-valued positive ideal solution) and PFSV-NIS (PFS-valued negative ideal solution), using

$$
\begin{aligned}
\text { PFSV-PIS } & =\left\{\ddot{\rho}_{1}^{+}, \ddot{\rho}_{2}^{+}, \ldots, \ddot{\rho}_{m}^{+}\right\} \\
& =\left\{\left(\vee_{k} \tau_{j k}, \wedge_{k} o_{j k}\right): k=1,2, \ldots, m\right\} \\
& =\left\{\left(\tau_{k}^{+}, o_{k}^{+}\right): k=1,2, \ldots, m\right\}
\end{aligned}
$$

and

$$
\begin{aligned}
\text { PFSV-NIS } & =\left\{\ddot{\rho}_{1}^{-}, \ddot{\rho}_{2}^{-}, \ldots, \ddot{\rho}_{m}^{-}\right\} \\
& =\left\{\left(\wedge_{k} \tau_{j k}, \vee_{k} o_{j k}\right): k=1,2, \ldots, m\right\} \\
& =\left\{\left(\tau_{k}^{-}, o_{k}^{-}\right): k=1,2, \ldots, m\right\}
\end{aligned}
$$

respectively, where $\vee$ represents PFS union and $\wedge$ denotes PFS intersection.

Step 7: Work out remoteness of every alternative from PFSV-PIS and PFSV-NIS, respectively, by making use of

$$
d_{j}^{+}=\sqrt{\Sigma_{k=1}^{m}\left\{\left(\tau_{j k}-\tau_{k}^{+}\right)^{2}+\left(o_{j k}-o_{k}^{+}\right)^{2}\right\}}
$$

and

$$
d_{j}^{-}=\sqrt{\Sigma_{k=1}^{m}\left\{\left(\tau_{j k}-\tau_{k}^{-}\right)^{2}+\left(o_{j k}-o_{k}^{-}\right)^{2}\right\}}
$$

for $j$ varying from 1 to $l$.

Step 8: Compute the relative closeness using

$$
\mathcal{C}_{j}^{*}=\frac{d_{j}^{-}}{d_{j}^{+}+d_{j}^{-}}
$$

Step 9: Prioritize the choices in descending (or ascending) array to have the preference order of all choices available.

Example 3.1 We apply Algorithm 1 in this example as follows: 
Step 1: Let $E=\left\{\mathcal{D}_{1}, \ldots, \mathcal{D}_{4}\right\}$ be the team comprising medical experts, $V=\left\{\ddot{\rho}_{i}: i=1, \ldots, 6\right\}$ the set of patients under study and $D=\left\{\eta_{i}: i=1, \ldots, 5\right\}$ the family of criteria, where

$\eta_{1}=$ vomiting

$\eta_{2}=$ jaundice,

$\eta_{3}=$ light/clay-colored stool,

$\eta_{4}=$ abdominal discomfort, and

$\eta_{5}=$ dark urine

Step 2: The matrix of weighted parameters $\mathcal{P}$ is

$$
\begin{aligned}
\mathcal{P} & =\left[w_{i j}\right]_{4 \times 5} \\
& =\left(\begin{array}{lllll}
S 1 & S 2 & S 0 & S 3 & S 3 \\
S 2 & S 3 & S 1 & S 2 & S 4 \\
S 1 & S 2 & S 1 & S 2 & S 0 \\
S 3 & S 4 & S 4 & S 3 & S 2
\end{array}\right) \\
& =\left(\begin{array}{lllll}
0.30 & 0.50 & 0.10 & 0.70 & 0.70 \\
0.50 & 0.70 & 0.30 & 0.50 & 0.90 \\
0.30 & 0.50 & 0.30 & 0.50 & 0.10 \\
0.70 & 0.90 & 0.90 & 0.70 & 0.50
\end{array}\right)
\end{aligned}
$$

where $w_{i j}$ represents the weight allocated by the team member $\mathcal{D}_{i}$ to the parameter $\eta_{j}$ keeping in view the phonological terms pre-decided in Table 2. In other words, the specialist $\mathcal{D}_{i}$ on the basis of different tests and visible condition of the patient assigns weight $w_{i j}$ to the parameter $\eta_{j}$. This weight, according to the specialist, assesses the stage/phase of hepatitis virus.

Step 3: The normalized weighted matrix, thus, comes out to be

$$
\begin{aligned}
\hat{N} & =\left[\hat{n}_{i j}\right]_{4 \times 5} \\
& =\left(\begin{array}{lllll}
0.31 & 0.37 & 0.10 & 0.58 & 0.56 \\
0.52 & 0.52 & 0.30 & 0.41 & 0.72 \\
0.31 & 0.37 & 0.30 & 0.41 & 0.08 \\
0.73 & 0.67 & 0.90 & 0.58 & 0.40
\end{array}\right)
\end{aligned}
$$

and hence the weight vector appears to be $\mathcal{W}=$ $(0.20,0.21,0.18,0.22,0.19)$.

Step 4: Assume that the four experts provide the following PFS matrices in which the PFN $(\tau, o)$ at the $(i, j)^{t h}$ position showing grades of patients row-wise and the symptoms/criteria column-wise.

$$
D_{1}=\left(\begin{array}{lllll}
(0.37,0.54) & (0.54,0.51) & (0.22,0.53) & (0.82,0.34) & (0.25,0.89) \\
(0.64,0.53) & (0.87,0.46) & (0.42,0.51) & (0.29,0.95) & (0.41,0.56) \\
(0.42,0.31) & (0.62,0.10) & (0.76,0.50) & (0.33,0.94) & (0.54,0.77) \\
(0.76,0.51) & (0.91,0.28) & (0.55,0.70) & (0.43,0.87) & (0.18,0.94) \\
(0.55,0.29) & (0.67,0.36) & (0.78,0.37) & (0.24,0.51) & (0.48,0.89) \\
(0.52,0.51) & (0.37,0.88) & (0.46,0.51) & (0.10,0.93) & (0.65,0.24)
\end{array}\right)
$$

$$
\begin{aligned}
D_{2}= & \left(\begin{array}{lllll}
(0.29,0.78) & (0.23,0.68) & (0.45,0.41) & (0.38,0.56) & (0.71,0.45) \\
(0.46,0.34) & (0.58,0.37) & (0.81,0.26) & (0.48,0.51) & (0.39,0.41) \\
(0.57,0.32) & (0.56,0.58) & (0.50,0.50) & (0.33,0.67) & (0.42,0.53) \\
(0.84,0.47) & (0.73,0.39) & (0.62,0.57) & (0.56,0.46) & (0.47,0.11) \\
(0.33,0.49) & (0.98,0.06) & (0.64,0.59) & (0.41,0.24) & (0.93,0.36) \\
(0.89,0.41) & (0.95,0.30) & (0.71,0.68) & (0.89,0.41) & (0.97,0.21)
\end{array}\right) \\
D_{3}= & \left(\begin{array}{lllll}
(0.33,0.66) & (0.39,0.60) & (0.34,0.47) & (0.60,0.45) & (0.48,0.67) \\
(0.55,0.44) & (0.73,0.42) & (0.62,0.39) & (0.39,0.73) & (0.40,0.49) \\
(0.50,0.32) & (0.59,0.34) & (0.63,0.50) & (0.33,0.81) & (0.48,0.65) \\
(0.80,0.49) & (0.82,0.34) & (0.59,0.64) & (0.50,0.67) & (0.33,0.53) \\
(0.12,0.35) & (0.71,0.15) & (0.87,0.13) & (0.56,0.29) & (0.67,0.49) \\
(0.45,0.13) & (0.89,0.12) & (0.68,0.19) & (0.47,0.42) & (0.32,0.19)
\end{array}\right)
\end{aligned}
$$$$
D_{4}=\left(\begin{array}{lllll}
(0.35,0.60) & (0.46,0.59) & (0.27,0.47) & (0.70,0.39) & (0.49,0.63) \\
(0.59,0.48) & (0.71,0.41) & (0.51,0.42) & (0.37,0.83) & (0.65,0.75) \\
(0.46,0.39) & (0.59,0.18) & (0.62,0.61) & (0.56,0.64) & (0.68,0.71) \\
(0.78,0.62) & (0.82,0.33) & (0.24,0.67) & (0.66,0.73) & (0.41,0.90) \\
(0.38,0.14) & (0.56,0.11) & (0.89,0.45) & (0.56,0.70) & (0.67,0.42) \\
(0.88,0.46) & (0.99,0.10) & (0.83,0.54) & (0.85,0.51) & (0.29,0.31)
\end{array}\right)
$$

Thus, the mean proportional matrix is

$$
\begin{aligned}
& A=\left(\begin{array}{lllll}
(0.33,0.64) & (0.64,0.59) & (0.31,0.47) & (0.60,0.43) & (0.45,0.64) \\
(0.56,0.44) & (0.72,0.41) & (0.57,0.38) & (0.38,0.74) & (0.45,0.54) \\
(0.48,0.33) & (0.59,0.24) & (0.62,0.53) & (0.38,0.76) & (0.52,0.66) \\
(0.79,0.52) & (0.82,0.33) & (0.47,0.64) & (0.53,0.67) & (0.33,0.47) \\
(0.30,0.29) & (0.71,0.14) & (0.79,0.34) & (0.42,0.40) & (0.67,0.51) \\
(0.65,0.33) & (0.75,0.24) & (0.66,0.43) & (0.43,0.53) & (0.49,0.23)
\end{array}\right) \\
& =\left[\dot{\rho}_{j k}\right]_{6 \times 5}
\end{aligned}
$$

Step 5: The weighted PFS matrix is

$$
\begin{aligned}
& B=\left(\begin{array}{lllll}
(0.07,0.13) & (0.14,0.13) & (0.05,0.08) & (0.13,0.09) & (0.09,0.12) \\
(0.11,0.09) & (0.15,0.09) & (0.10,0.07) & (0.08,0.16) & (0.09,0.10) \\
(0.10,0.07) & (0.13,0.05) & (0.11,0.09) & (0.08,0.16) & (0.10,0.13) \\
(0.16,0.11) & (0.17,0.07) & (0.08,0.11) & (0.11,0.14) & (0.06,0.09) \\
(0.06,0.06) & (0.15,0.03) & (0.14,0.06) & (0.09,0.09) & (0.13,0.10) \\
(0.13,0.07) & (0.16,0.05) & (0.12,0.08) & (0.09,0.11) & (0.09,0.04)
\end{array}\right) \\
& =\left[\ddot{\rho}_{j k}\right]_{6 \times 5}
\end{aligned}
$$

where $\ddot{\rho}_{j k}=\mathfrak{w}_{k} \times \dot{\rho}_{j k}$.

Step 6: Thus, PFSV-PIS and PFSV-NIS, respectively, are

$$
\begin{aligned}
\text { PFSV-PIS }= & \left\{\ddot{\rho}_{1}^{+}, \ldots, \ddot{\rho}_{5}^{+}\right\} \\
= & \{(0.16,0.06),(0.17,0.03),(0.14,0.06), \\
& (0.13,0.09),(0.13,0.04)\}
\end{aligned}
$$

and

$$
\begin{aligned}
\text { PFSV-NIS }= & \left\{\ddot{\rho}_{1}^{-}, \ldots, \ddot{\rho}_{5}^{-}\right\} \\
= & \{(0.06,0.13),(0.13,0.13),(0.05,0.11), \\
& (0.08,0.16),(0.06,0.13)\}
\end{aligned}
$$

Step 7, 8: The Euclidean distances of each patient from PFSV-PIS and PFSV-NIS and corresponding relative coefficients of closeness are given in Table 3 .

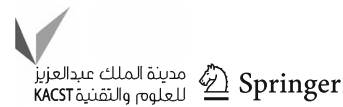


Table 3 Distance and coefficient of closeness of each patient

\begin{tabular}{llll}
\hline Patient $\left(\ddot{\rho}_{i}\right)$ & $d_{i}^{+}$ & $d_{i}^{-}$ & $\mathcal{C}_{i}^{*}$ \\
\hline$\ddot{\rho}_{1}$ & 0.2010 & 0.0975 & 0.3266 \\
$\ddot{\rho}_{2}$ & 0.1473 & 0.1095 & 0.4264 \\
$\ddot{\rho}_{3}$ & 0.1546 & 0.1311 & 0.4589 \\
$\ddot{\rho}_{4}$ & 0.1432 & 0.1393 & 0.4931 \\
$\ddot{\rho}_{5}$ & 0.1249 & 0.1916 & 0.6054 \\
$\ddot{\rho}_{6}$ & 0.0768 & 0.1822 & 0.7035 \\
\hline
\end{tabular}

Step 9: Thus, the preference order of the patients is

$$
\ddot{\rho}_{6} \succ \ddot{\rho}_{5} \succ \ddot{\rho}_{4} \succ \ddot{\rho}_{3} \succ \ddot{\rho}_{2} \succ \ddot{\rho}_{1}
$$

This ranking is depicted in Fig. 4.

This ranking advocates that the patient $\ddot{\rho}_{6}$ is in more critical situation.

\section{Medical diagnosis using PFS VIKOR}

The word VIKOR is abbreviated version of "Vlse Kriterijumska Optimizacija Kompromisno Resenje" which comes from Serbian language and is used for multiple criteria analysis. VIKOR was primarily recognized by Serafim Opricovic to handle DMPs equipped with non-commensurable and acrimonious criteria, with the assumption that finding the middle ground is apposite for conflict determination. It was kept in mind that the decision makers usually search for such a solution that is neighboring to the ideal, and that the alternatives are examined keeping in view all standard moralities. This technique has arisen as a quite widely held MCDM technique mainly due to its solution meticulousness and computational comfort. It prominences on determining on and grading from viable choices, and picks compromise solution for a problem having uneven standards to ease the decision makers in accomplishing an unbiased final judgment. It decides the compromise ranking list recognized for unambiguous degree of nearness to the ideal solution.

We initiate by elucidating the suggested technique inch by inch. The first six steps are same as cited in Algorithm 1 for PFS TOPSIS method, so we skip them. We elaborate the remnant steps as follows:

\section{Algorithm 2 (PFS VIKOR)}

Step 7: Work out the strategic values of VIKOR for each choice the group utility value $S_{i}$, the individual regret value $R_{i}$, and compromise value $Q_{i}$ using

$$
\begin{aligned}
S_{i} & =\Sigma_{j=1}^{m} \mathfrak{w}_{j}\left(\frac{d\left(\ddot{\rho}_{j}^{+}, \ddot{\rho}_{i j}\right)}{d\left(\ddot{\rho}_{j}^{+}, \ddot{\rho}_{j}^{-}\right)}\right) \\
R_{i} & =\max _{j=1}^{m} \mathfrak{w}_{j}\left(\frac{d\left(\ddot{\rho}_{j}^{+}, \ddot{\rho}_{i j}\right)}{d\left(\ddot{\rho}_{j}^{+}, \ddot{\rho}_{j}^{-}\right)}\right) \\
Q_{i} & =\kappa\left(\frac{S_{i}-S^{-}}{S^{+}-S^{-}}\right)+(1-\kappa)\left(\frac{R_{i}-R^{-}}{R^{+}-R^{-}}\right)
\end{aligned}
$$

where $S^{+}=\max _{i} S_{i}, S^{-}=\min _{i} S_{i}, R^{+}=$ $\max _{i} R_{i}$, and $R^{-}=\min _{i} R_{i}$. The real number $\kappa$ is termed as coefficient of decision mechanism. The role of the coefficient $\kappa$ is that if compromise solution is to be selected by majority, we choose $\kappa>0.5$; for consensus we use $\kappa=0.5$, and $\kappa<0.5$ represents veto. $\mathfrak{w}_{j}$ represents the weight of the $j^{\text {th }}$ criteria, which expresses its relative importance.

Step 8: Arrange $S_{i}, R_{i}$, and $Q_{i}$ in ascending array to establish three lists of rankings containing ranking list of $S_{i}, R_{i}$, and that of $Q_{i}$. The choice $\ddot{\rho}_{\alpha}$ would be nominated as compromise solution provided it holds the supreme position in $Q_{i}$ (having minimum value) and gratifies the following couple of conditions at the same time:

C-1 Tolerable benefit:

If $\ddot{\rho}_{\alpha_{1}}$ and $\ddot{\rho}_{\alpha_{2}}$ are two top most choices in $Q_{i}$, then

$$
Q\left(\ddot{\rho}_{\alpha_{2}}\right)-Q\left(\ddot{\rho}_{\alpha_{1}}\right) \geq \frac{1}{n-1}
$$

where the positive integer $n$ represents the number of parameters.

C-2 Tolerable permanence:

The choice $\ddot{\rho}_{\alpha_{1}}$ must be top ranked by at least one of $S_{i}$ and $R_{i}$.

If these two conditions are not satisfied at the same time, then we are led to the existence of manifold compromise solutions. In such a scenario, the following conditions will be activated:

(i) If merely $C$-1 is gratified, then both alternatives $\ddot{\rho}_{\alpha_{1}}$ and $\ddot{\rho}_{\alpha_{2}}$ will serve as the compromise solutions.

(ii) If, however, $C$ - 1 is not gratified and

$$
Q\left(\ddot{\rho}_{\alpha_{k}}\right)-Q\left(\ddot{\rho}_{\alpha_{1}}\right) \geq \frac{1}{n-1}
$$

then the alternatives $\ddot{\rho}_{\alpha_{1}}, \ddot{\rho}_{\alpha_{2}}, \ldots, \ddot{\rho}_{\alpha_{k}}$ would serve as the multiple compromise solutions.

Example 4.1 We re-attempt Example 3.1 using VIKOR elucidated in Algorithm 2. 
Fig. 4 Column chart showing ranking of patients

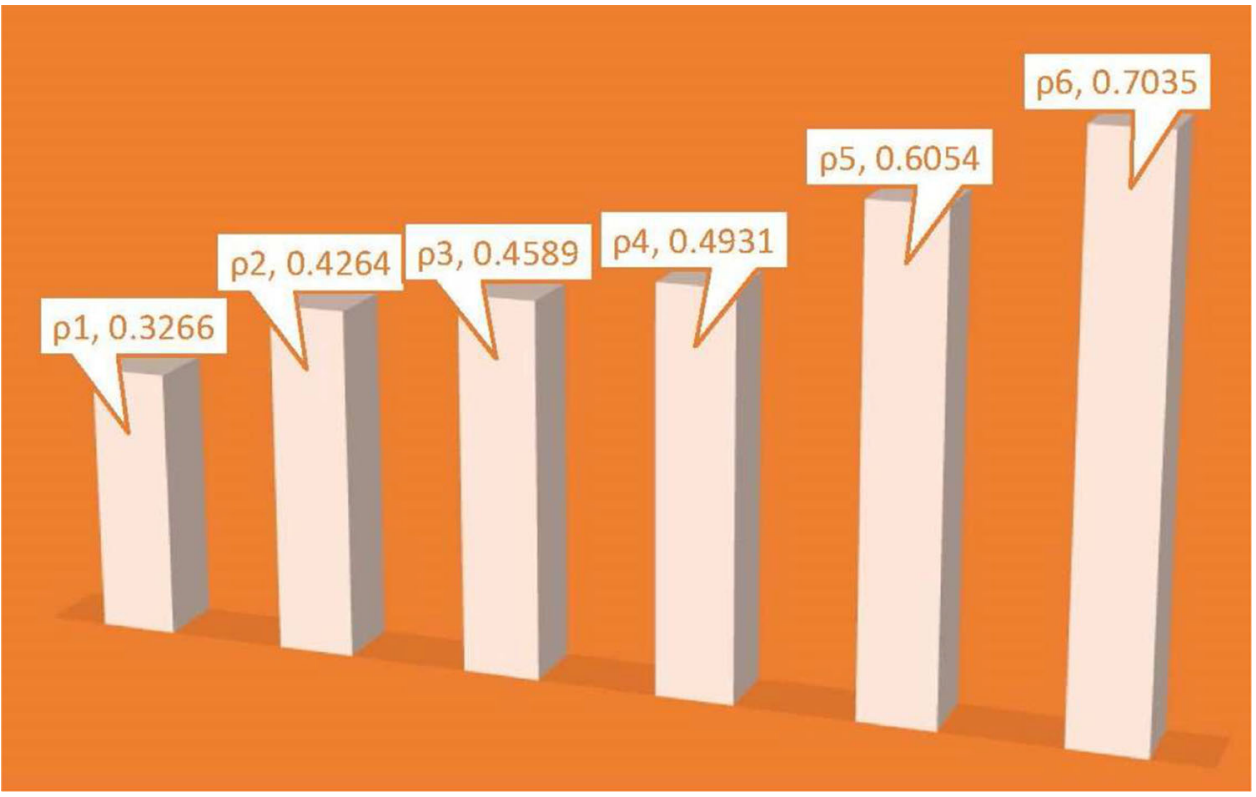

Table 4 Values of $S_{i}, R_{i}$ and $Q_{i}$ for each patient

\begin{tabular}{llll}
\hline Patient $\left(\ddot{\rho}_{i}\right)$ & $S_{i}$ & $R_{i}$ & $Q_{i}$ \\
\hline$\ddot{\rho}_{1}$ & 0.7004 & 0.2036 & 0.9232 \\
$\ddot{\rho}_{2}$ & 0.6910 & 0.2200 & 0.9882 \\
$\ddot{\rho}_{3}$ & 0.6391 & 0.2200 & 0.9188 \\
$\ddot{\rho}_{4}$ & 0.5776 & 0.1433 & 0.4738 \\
$\ddot{\rho}_{5}$ & 0.4051 & 0.1638 & 0.3403 \\
$\ddot{\rho}_{6}$ & 0.3260 & 0.1143 & 0.0004 \\
\hline
\end{tabular}

Step 7: Choosing $\kappa=0.5$, the values of $S_{i}, R_{i}$, and $Q_{i}$ for each patient are computed using

$$
\begin{aligned}
S_{i} & =\Sigma_{j=1}^{5} \mathfrak{w}_{j}\left(\frac{d\left(\ddot{\rho}_{j}^{+}, \ddot{\rho}_{i j}\right)}{d\left(\ddot{\rho}_{j}^{+}, \ddot{\rho}_{j}^{-}\right)}\right) \\
R_{i} & =\max _{j=1}^{5} \mathfrak{w}_{j}\left(\frac{d\left(\ddot{\rho}_{j}^{+}, \ddot{\rho}_{i j}\right)}{d\left(\ddot{\rho}_{j}^{+}, \ddot{\rho}_{j}^{-}\right)}\right) \\
Q_{i} & =\kappa\left(\frac{S_{i}-S^{-}}{S^{+}-S^{-}}\right)+(1-\kappa)\left(\frac{R_{i}-R^{-}}{R^{+}-R^{-}}\right)
\end{aligned}
$$

and are given in Table 4.

Step 8: The rank of choices is as under:

$$
\begin{aligned}
\text { By } Q_{i}: & \ddot{\rho}_{6} \prec \ddot{\rho}_{5} \prec \ddot{\rho}_{4} \prec \ddot{\rho}_{3} \prec \ddot{\rho}_{1} \prec \ddot{\rho}_{2} \\
\text { By } S_{i}: & \ddot{\rho}_{6} \prec \ddot{\rho}_{5} \prec \ddot{\rho}_{4} \prec \ddot{\rho}_{3} \prec \ddot{\rho}_{2} \prec \ddot{\rho}_{1} \\
\text { By } R_{i}: & \ddot{\rho}_{6} \prec \ddot{\rho}_{4} \prec \ddot{\rho}_{5} \prec \ddot{\rho}_{1} \prec \ddot{\rho}_{2}=\ddot{\rho}_{3}
\end{aligned}
$$

Since

$$
Q\left(\ddot{\rho}_{5}\right)-Q\left(\ddot{\rho}_{6}\right)=0.3399 \geq \frac{1}{4}
$$

and $\ddot{\rho}_{6}$ is also best ranked by $S_{i}$ and/or $R_{i}$, so we conclude that the patient $\ddot{\rho}_{6}$ is in more critical situation as compared to the other five ones.

The rankings of patients w.r.t. $Q_{i}, R_{i}$ and $S_{i}$ are depicted in Fig. 5.

\section{Medical diagnosis by generalized PFS aggregation operators}

In this unit, we first generalize the PFS aggregation operators to fit our problem. The first five steps are same as Algorithm 1. Therefore, we skip them and begin with step 6 .

Algorithm 3 (Generalized PFS aggregation operators method)

Step 6: Find the cardinal matrix $M_{c(B)}=\left[\frac{1}{l} \sum_{j=1}^{l} \dot{\rho}_{j k}: k=\right.$ $1,2, \ldots, m]_{1 \times m}$

Step 7: Compute the aggregated PF matrix $B^{*}$ using $B^{*}=$ $\frac{B \times M_{c(B)}^{t}}{|D|}$.

Step 8: Work out the values of score function using $s=$ $\tau^{2}-o^{2}$. The optimal choice is the alternative with highest value of $s$.

Example 5.1 We, once again, attempt Example 3.1 using generalized PFS aggregation operators method elucidated in Algorithm 3. 
Fig. 5 Multiple bar chart of rankings

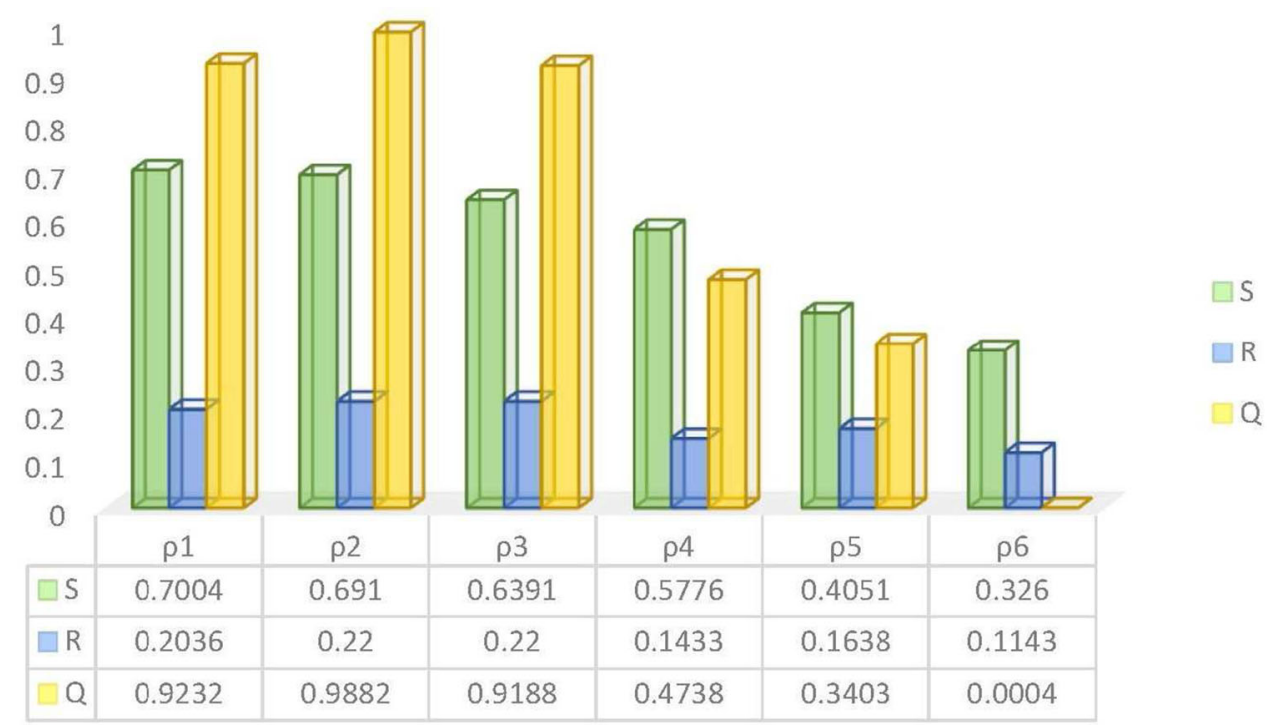

Step 6: The cardinal matrix is

$$
M_{\mathrm{c}(B)}=\left[\begin{array}{ll}
(0.105,0.088) & (0.150,0.070)(0.100,0.082) \\
(0.097,0.125) & (0.093,0.097)
\end{array}\right]
$$

Step 7: The aggregated PF matrix is

$$
\begin{aligned}
B^{*}= & \frac{B \times M_{c(B)}^{t}}{|E|} \\
= & \left.\begin{array}{lllll}
(0.07,0.13) & (0.14,0.13) & (0.05,0.08) & (0.13,0.09) & (0.09,0.12) \\
(0.11,0.09) & (0.15,0.09) & (0.10,0.07) & (0.08,0.16) & (0.09,0.10) \\
(0.10,0.07) & (0.13,0.05) & (0.11,0.09) & (0.08,0.16) & (0.10,0.13) \\
(0.16,0.11) & (0.17,0.07) & (0.08,0.11) & (0.11,0.14) & (0.06,0.09) \\
(0.06,0.06) & (0.15,0.03) & (0.14,0.06) & (0.09,0.09) & (0.13,0.10) \\
(0.13,0.07) & (0.16,0.05) & (0.12,0.08) & (0.09,0.11) & (0.09,0.04)
\end{array}\right) \\
= & \left(\begin{array}{l}
(0.105,0.088) \\
(0.150,0.070) \\
(0.100,0.082) \\
(0.097,0.125) \\
(0.093,0.097)
\end{array}\right) \\
& \left(\begin{array}{l}
(0.0109,0.0100) \\
(0.0120,0.0099) \\
(0.0116,0.0099) \\
(0.0133,0.0100) \\
(0.0127,0.0067) \\
(0.0134,0.0068)
\end{array}\right)
\end{aligned}
$$

Step 8: The values of score function for each patient are worked out in Table 5.

Thus, the ranking of patients is

$\ddot{\rho}_{6} \succ \ddot{\rho}_{5} \succ \ddot{\rho}_{4} \succ \ddot{\rho}_{2} \succ \ddot{\rho}_{3} \succ \ddot{\rho}_{1}$

This ranking is portrayed in Fig. 6 .
Table 5 Score values for patients

\section{Comparison of three methods: commentary}

The rankings of patients made through TOPSIS, VIKOR and generalized PFS aggregation operator methods are depicted in Fig. 7. To make the comparison conceivable, we have drawn the values of $1-Q$ instead of $Q$ in VIKOR. Moreover, to make the columns for score values visible, we have scaled the score values by multiplying them with 1000 . The first series on the left shows TOPSIS, second VIKOR and the third one is used for scaled score values.

We observe that the optimal choice by all the three techniques is the same, i.e., $\ddot{\rho}_{6}$. In TOPSIS, there is only one check that the optimal solution must be neighboring to the positive ideal solution and most distant from the negative ideal solution. In VIKOR, there are more than one check points. For example, we make use of the values of $Q_{i}, R_{i}$ and $S_{i}$ to test acceptable advantage and acceptable stability. Thus, if a weak solution succeeds in passing through one check, it will be rejected in next check point. In VIKOR, there is facility of more than one compromise solutions.

In TOPSIS we use the grade index containing distances from PIS and NIS. The distances so computed are simply added without taking account of their virtual prominence. The distance automatically could characterize some equilib- 
Fig. 6 Ranking of patients

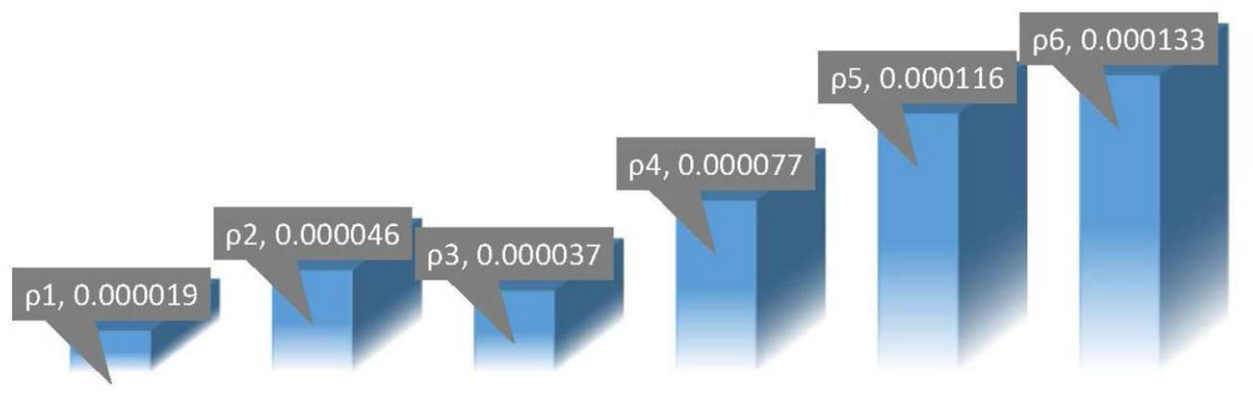

Fig. 7 Ranking comparison obtained through TOPSIS, VIKOR and generalized PFS aggregation operator methods

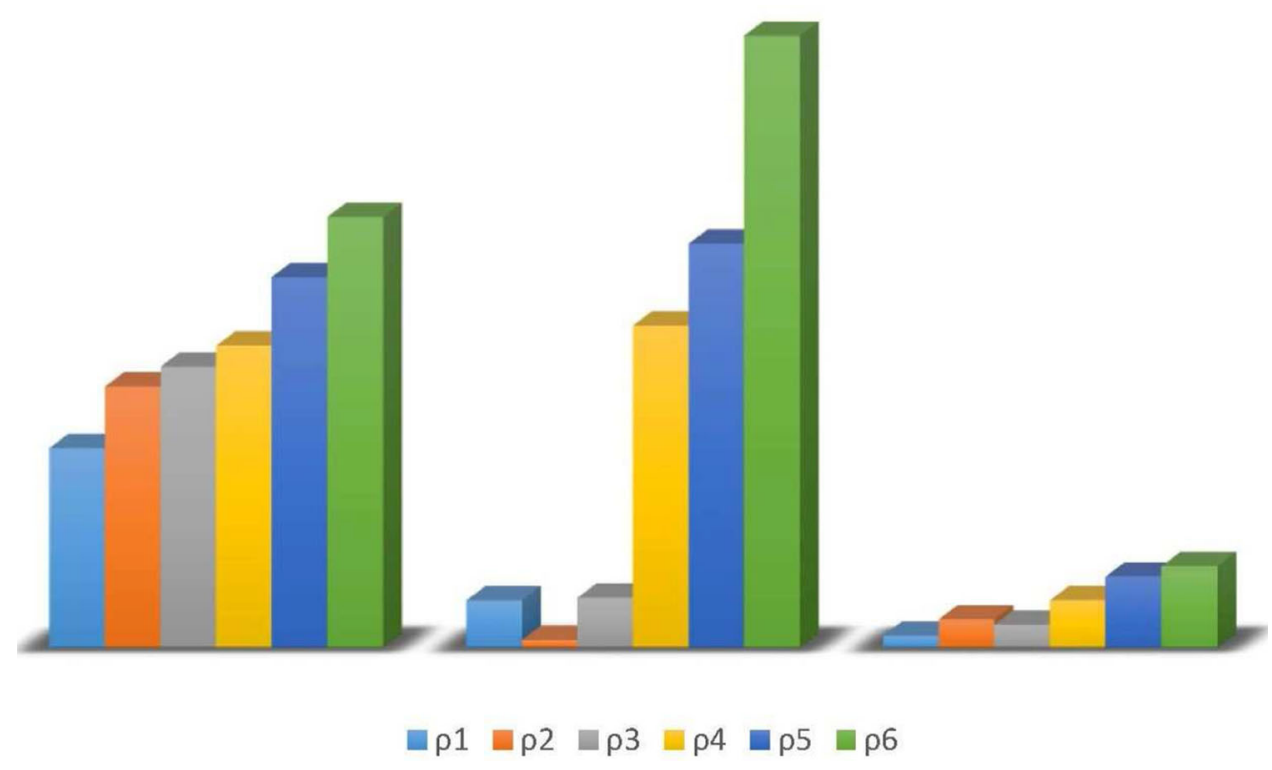

rium among entire and separate contentment, but practices it in a changed manner as in VIKOR. In VIKOR, the weight $\kappa$ is familiarized. Both approaches arrange for a ranking grade. The premier ranked choice attained by VIKOR is neighboring to ideal solution. Nevertheless, the top ranked choice determined by TOPSIS is paramount regarding ranking table. This does not imply that it is at all times contiguous to the supreme solution. Apart from ranking, VIKOR gives the facility of having a compromise solution endowed with an improvement (advantage) level.

On the other hand, the method of generalized PFS aggregation operators is easy to manipulate and offers more computational ease as compared to the other two techniques. In view of this discussion, we may conclude that VIKOR model has an edge over TOPSIS and yields more reliable outputs. However, keeping in view the computational ease, the method of generalized PFS aggregation operators is most preferable. It depends upon the problem under study that how much precision we desire. Depending upon the level of accuracy required, we choose the method.

\section{Comparison analysis and superiority of proposed work}

We observe that the optimal solution remains the same by use of either of the three algorithms in this article. Further, the technique suggested in this article are facile to apply and yield definitive outputs. The comparison of final rankings with some existing methodologies is given in Table 6 .

\section{Conclusion}

We proposed three algorithms, i.e., PFS TOPSIS, VIKOR and generalized PFS aggregation operators method, for modeling uncertainties in MCGDM problem from health management using PFSSs. The proposed Algorithms have been efficaciously applied on ranking different patients. Brief but comprehensive detail of different types of hepatitis along with symptoms of these types are also brought under discussion. To comprehend the final rankings, we have made use of 
Table 6 Comparison of proposed techniques with some exiting methodologies

\begin{tabular}{ll}
\hline Methodology & Optimal choice \\
\hline Algorithm 1, 2, 3 (Proposed) & $\ddot{\rho}_{6}$ \\
Pythagorean fuzzy aggregation operators (Peng and Yuan [24]) & $\ddot{\rho}_{6}$ \\
Prioritized weighted aggregation operators (Liu et al. [15]) & $\ddot{\rho}_{6}$ \\
Generalized intuitionistic fuzzy soft power aggregation operator (Garg and Arora [9]) & $\ddot{\rho}_{6}$ \\
Intuitionistic fuzzy aggregation operators (Xu [28]) & $\ddot{\rho}_{6}$ \\
\hline
\end{tabular}

statistical charts. Comparison of three rankings along with solid argument about more feasible method is also talked over. We have made comparison between the ultimate gradings generated through the three models with the assistance of statistical chart.

The proposed model has tremendous potential for further exploration in theoretical besides application perspective and may be efficiently applied in other hybrid structures of fuzzy sets with slight amendments. The idea may be efficiently employed in handling uncertainties in different domains of real life situations including energy management, business, artificial intelligence, chemical engineering, marketing, image processing, electoral system, logistics, pattern recognition, machine learning, manufacturing, medical diagnosis, trade analysis, environment management, game theory, forecasting, robotics, coding theory and recruitment problems.

Author Contributions The authors contributed to each part of this paper equally. The authors read and approved the final manuscript.

\section{Declarations}

Conflict of interest The authors declare that they have no conflict of interest.

Open Access This article is licensed under a Creative Commons Attribution 4.0 International License, which permits use, sharing, adaptation, distribution and reproduction in any medium or format, as long as you give appropriate credit to the original author(s) and the source, provide a link to the Creative Commons licence, and indicate if changes were made. The images or other third party material in this article are included in the article's Creative Commons licence, unless indicated otherwise in a credit line to the material. If material is not included in the article's Creative Commons licence and your intended use is not permitted by statutory regulation or exceeds the permitted use, you will need to obtain permission directly from the copyright holder. To view a copy of this licence, visit http://creativecomm ons.org/licenses/by/4.0/.

\section{References}

1. Atanassov K (1984) Intuitionistic fuzzy sets. In: Sgurev V (ed) VII ITKRs Session, Sofia, June 1983. Central Sci. and Techn. Library, Bulg. Academy of Sciences
2. Atanassov K, Stoeva S (1983) Intuitionistic fuzzy sets. In: Polish symp. on interval and fuzzy mathematics, Poznan, pp 23-26

3. Atanassov KT (1986) Intuitionistic fuzzy sets. Fuzzy Sets Syst 20:87-96

4. Atanassovc KT (1999) Intuitionistic fuzzy sets: theory and application. Studies in fuzziness and soft computing, vol XVIII. Physica Verlag, Heidelberg, pp 324-330

5. Çă̆man N, Enginoglu S, Çitak F (2011) Fuzzy soft set theory and its applications. Iran J Fuzzy Syst 8(8):137-147

6. Davvaz B, Sadrabadi EH (2016) An application of intuitionistic fuzzy sets in medicine. Int J Biomath 9(3):1650037-1-165003715

7. Dikshit-Ratnaparkhi A, Bormane D, Ghongade R (2020) A novel entropy-based weighted attribute selection in enhanced multicriteria decision-making using fuzzy TOPSIS model for hesitant fuzzy rough environment. Complex Intell Syst. https://doi.org/10.1007/ s40747-020-00187-8

8. Eraslan S, Karaaslan F (2015) A group decision making method based on TOPSIS under fuzzy soft environment. J New Theory 3:30-40

9. Garg H, Arora R (2019) Generalized intuitionistic fuzzy soft power aggregation operator based on t-norm and their application in multicriteria decision-making. Int J Intell Syst 34(2):215-246

10. Garg H, Arora R (2018) Dual hesitant fuzzy soft aggregation operators and their application in decision-making. Cognit Comput 10(5):769-789

11. Garg H, Arora R (2018) A nonlinear-programming methodology for multi-attribute decision-making problem with interval-valued intuitionistic fuzzy soft sets information. Appl Intell 48(8):20312046

12. Guleria A, Bajaj RK (2019) On Pythagorean fuzzy soft matrices, operations and their applications in decision making and medical diagnosis. Soft Comput 23(17):7889-7900

13. Hwang CL, Yoon K (1981) Multiple attribute decision makingmethods and applications. Springer, Heidelberg

14. Kalkan S, Turanli M, Özden Ü, Başar Ö (2017) Comparison of ranking results obtained by TOPSIS and VIKOR methods, using the same criteria as Times Higher Education World University ranking. Eur J Bus Soc Sci 6(1):107-122

15. Liu P, Akram M, Sattar A (2020) Extensions of prioritized weighted aggregation operators for decision-making under complex q-rung orthopair fuzzy information. J Intell Fuzzy Syst. https://doi.org/10. 3233/JIFS-200789

16. Maji PK, Roy AR, Biswas R (2001) Intuitionistic fuzzy soft sets. J Fuzzy Math 9(3):677-692

17. Molodtsov D (1999) Soft set theory-first results. Comput Math Appl 37(4-5):19-31

18. Naeem K, Riaz M, Karaaslan F (2021) Some novel features of Pythagorean $m$-polar fuzzy sets with applications. Complex Intell Syst 7:459-475 
19. Naeem K, Riaz M, Peng XD, Afzal D (2019) Pythagorean fuzzy soft MCGDM methods based on TOPSIS, VIKOR and aggregation operators. J Intell Fuzzy Syst 37(5):6937-6957

20. Naeem K, Riaz M, Afzal D (2019) Pythagorean $m$-polar fuzzy sets and TOPSIS method for the selection of advertisement mode. J Intell Fuzzy Syst 37(6):8441-8458

21. Opricovic S, Tzeng HG (2004) Compromise solution by MCDM methods: a comparative analysis of VIKOR and TOPSIS. Eur J Oper Res 156:445-455

22. Opricovic S, Tzeng HG (2007) Extended VIKOR method in comparison with other outranking methods. Eur J Oper Res 178(2):514-529

23. Peng XD, Yang YY, Song J, Jiang Y (2015) Pythagorean fuzzy soft set and its application. Comput Eng 41(7):224-229

24. Peng XD, Yuan HY (2016) Fundamental properties of Pythagorean fuzzy aggregation operators. Fundamenta Informaticae 147(4):415-446

25. Salabun W, Watrobski J, Shekhovtsov A (2020) Are MCDA methods benchmarkable? A comparative study of TOPSIS, VIKOR, COPRAS, and PROMETHEE II methods. Symmetry 12(9):1549

26. Shekhovtsov A, Salabun W (2020) A comparative case study of the VIKOR and TOPSIS rankings similarity. Proc Comput Sci 176:3730-3740. https://doi.org/10.1016/j.procs.2020.09.014
27. Wang YM, Elhag TMS (2006) Fuzzy TOPSIS method based on alpha level sets with an application to bridge risk assessment. Expert Syst Appl 31(2):309-319

28. Xu ZS (2007) Intuitionistic fuzzy aggregation operators. IEEE Trans Fuzzy Syst 15(6):1179-1187

29. Yager RR (2013) Pythagorean fuzzy subsets. In: IFSA World Congress and NAFIPS Annual Meeting (IFSA/NAFIPS), 2013 Joint. IEEE, Edmonton, Canada, pp 57-61

30. Yager RR, Abbasov AM (2013) Pythagorean membership grades, complex numbers, and decision making. Int J Intell Syst 28(5):436452

31. Yager RR (2014) Pythagorean membership grades in multi-criteria decision making. IEEE Trans Fuzzy Syst 22(4):958-965

32. Zadeh LA (1965) Fuzzy sets. Inf Control 8:338-356

33. Zhang X, Xu Z (2014) Extension of TOPSIS to multiple criteria decision making with Pythagorean fuzzy sets. Int J Intell Syst 29(12):1061-1078

Publisher's Note Springer Nature remains neutral with regard to jurisdictional claims in published maps and institutional affiliations. 